\title{
Where do the Progenitors of Millisecond Pulsars come from?
}

\author{
Ali Taani ${ }^{1, \star}$, Chengmin Zhang ${ }^{1}$, Mashhoor Al-Wardat ${ }^{2}$, and Yongheng Zhao ${ }^{1}$ \\ 1 National Astronomical Observatories, Chinese Academy of Sciences, Beijing 100012, China \\ 2 Department of Physics, Al-Hussein Bin Talal University, P.O.Box 20, 71111, Ma'an, Jordan
}

Received 30 May 2005, accepted 11 Nov 2005

Published online later

Key words pulsars: general, stars: neutron stars, white dwarfs, cataclysmic variables, $\mathrm{x}$-ray binaries.

\begin{abstract}
Observations of a large population of Millisecond Pulsars (MSPs) show a wide divergence in the orbital periods (from approximately hours to a few months). In the standard view, Low-Mass X-Ray Binaries (LMXBs) are considered as progenitors for some MSPs during the recycling process. We present a systematic study that combines different types of compact objects in binaries such as Cataclysmic Variables (CVs), LMXBs and MSPs. We plot them together in the so called Corbet diagram. Larger and different samples are needed to better constrain the result as a function of the environment and formations. A scale diagram showing the distribution of MSPs for different orbital periods and the aspects for their progenitors relying on Accretion Induced Collapse (AIC) of white dwarfs in binaries. Thus massive CVs $\left(M \geq 1.1 M_{\odot}\right)$ can play a vital role on binary evolution, as well as of the physical processes involved in the formation and evolution of neutron stars and their magnetic fields, and could turn into binary MSPs with different scales of orbital periods; this effect can be explained by the AIC process. This scenario also suggests that some fraction of isolated MSPs in the Galactic disk could be formed through the same channel, formingthe contribution of some CVs to the single-degenerate progenitors of Type Ia supernova. Furthermore, we have refined the statistical distribution and evolution by using updated data. This implies that the significant studies of compact objects in binary systems can benefit from the Corbet diagram.
\end{abstract}

(C) 2006 WILEY-VCH Verlag GmbH \& Co. KGaA, Weinheim

\section{Introduction}

For many years, studies of the population of millisecond pulsars (MSPs) have been plagued by small-number statistics in order to obtain a more qualitative inspection of their progenitors. In a binary system the mass of the Neutron Star (NS) is larger than the mass of its companion (Bhattacharya \& van den Heuvel 1991), which causes it to have a short period (a few hours to days). However, it should be noted that $20 \%$ of MSPs are isolated (Camilo et al. 2001), but it is not clear how they have lost their presumed past companions. A widely held view is that the isolated cases are results of the primary's Super Nova (SN) type Ia explosions, which would have caused the loss of their companions by either evaporation or kicking out of the system (Bhattacharya 1996; Ferrario \& Wickramasinghe 2007ab).

Several statistical studies have questioned whether the known low-mass X-ray binary (LMXB) populations could produce the observed MSPs (e.g. Alpar et al. 1982; Srinivasan \& van den Heuvel 1982; Burderi et al. 2007; Liu et al. 2011 ). In the accepted scenario of this evolution, an old and quiescent NS is spun up to millisecond periods by the accretion of matter from its companion, during the LMXB phase of evolution. All of the previously mentioned papers, however, focused on systems in short orbital periods.

Moreover, Accretion Induced Collapse (AIC) of white dwarfs (WDs) has been proposed as an alternative source of

\footnotetext{
* Corresponding author: e-mail: alitaani@bao.ac.cn
}

recycled pulsars sufficient to obviate the difficulties with the standard model (van den Heuvel 2004; Hurley et al. 2010).

While other authors have questioned the viability of an AIC origin for the recycled pulsars on theoretical and statistical grounds (van den Romani 1990; Heuvel 2004). Ferrario \& Wickramasinghe (2007ab) argued that the AIC channel can form binary MSPs of all of observable types. Champion et al. (2008) proposed that the AIC could produce the observed orbital parameters of PSR J1903+0327. This scenario has a number of attractive features and it is desirable to have quantitative tests of expected results. This study aimed to investigate the binary MSPs and their progenitors within different binaries with compact companions from different catalogues. Furthermore we are paying particular attention to the AIC process in the observed sample of CVs.

However, there are several numerical simulation models that employ different physical mechanisms to model an AIC process. Dessart et al. (2007) have performed 2D multigroup neutrino-transport MHD simulations. Also Abdikamalov et al. (2010) have presented results from an extensive set of general-relativistic AIC simulations using a microphysical finite-temperature equation of state during collapse; they investigated a set of 114 progenitor models in axisymmetric rotational equilibrium, with a wide range of rotational configurations, temperatures and central densities, and resulting WD masses. In addition, Liu et al. (2011) have studied the Corbet diagram with regard to Be/X-ray binaries, and they investigated the progenitors of IGR J184830311 and IGR J11215-5952. Yan et al. (2010) studied the 


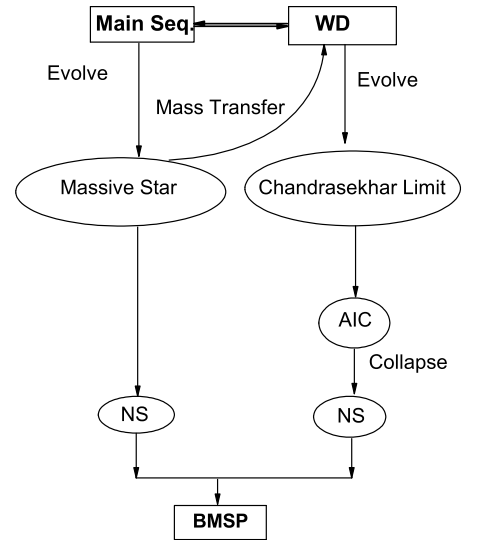

Fig. 1 The flow-chart illustrates the AIC evolutionary scenario of MSPs.

correlation between LMXBs and normal pulsars in the Corbet diagram.

Progress towards the current sample led to a resurgence of discoveries and interest from observers and theorists concerning the new distribution of MSPs. The mechanisms responsible for their formation in Globular Clusters and Galactic disks are different (Sutantyo \& Li 2000; Ivanova et al. 2008). Consequently, we show that from studying the Corbet-diagram (correlation of spin period vs. orbital period diagram) one is able to obtain clues about how the evolution of MSPs differs from those in binary and isolated systems (Yan et al. 2010).

This paper is motivated by previous work investigating the statistical properties of binary MSPs and their progenitors with long orbital periods (a few months). We defer further discussion on the model until the data collection is completed. The outline of this paper is as follows: In section 2, we review the formation routes including the recycling process and AIC of a white dwarf. In section 3, we discuss the Corbet diagram. We present the data acquisition and target selection in sections 4. Summary and conclusions are presented in section 5 .

\section{The Formation Routes for MSPs}

Interaction between the NS and its companion star is clearly important in determining the activity of any particular system. In order to understand this interaction and the consequent activity, we shall first discuss the formation scenarios in which the MSPs are formed, beginning with the standard route of the recycling process. On the other hand, we shall also discuss an alternative scenario by AIC of WDs.

\subsection{Fast-spinning "Recycling Process"}

The standard evolutionary scenario in which Binary MSPs are formed from LMXBs is considered as a powerful tools for the study of orbital kinematics in binary MSPs systems.

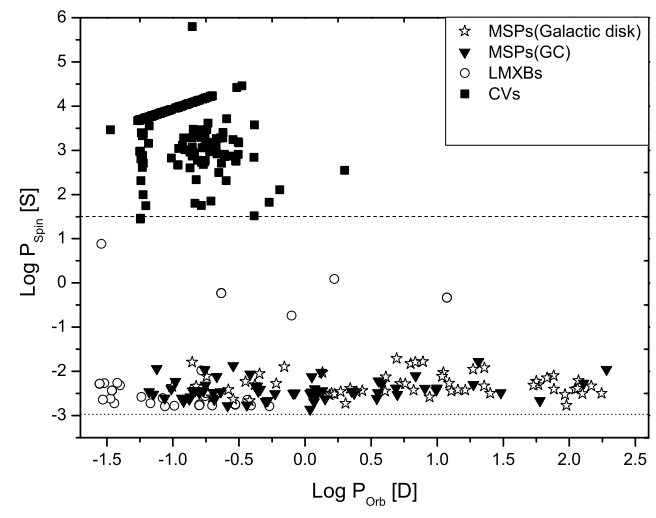

Fig. 2 The spin period vs. the orbital periods for binaries (Corbet diagram). Different symbols denote different types of binary systems. Here the CVs are represented (black squares), LMXBs (white circles), MSPs in the Galactic disk (white stars) and MSPs in globular cluster (black triangles). The horizontal dashed line at a spin period of 30 s splits the figure into two parts; WDs with $P>30 s$ and NSs with $P<30$ s. Dotted line represents the spin period at $1 \mathrm{~ms}$.

The evolutionary path is briefly described as follows: A highfield ( $B \sim 10^{12}-10^{13} \mathrm{G}$ ), rapidly rotating NS is born in a binary with a low-mass ( $1 M_{\odot}$ ) main-sequence companion star. During the SN that produces the NS, mass loss and kick imparted on the NS cause the orbit to be eccentric. This NS remains bound to its companion and spins down like a normal pulsar for the next $10^{6}$ to $10^{7}$ yrs (Lyne et al. 2004; Lorimer 2009), until passing the so-called "death line" in the magnetic field-spin period (BP) diagram. As the companion evolves, it eventually fills its Roche lobe. At this stage, transfer of matter from the companion to the NS creates an X-ray binary and tidal friction serves to circularize the orbit.

Mass accretion onto the NS gives rise to X-ray emission and induced magnetic field decay (the mechanisms for the field decay induced by accretion are, however, not well understood), therefore it spins up to a millisecond period. As a result, the systems can be observed as LMXBs, which generate brighter shorter lived sources. (Bhattacharya \& van den Heuvel 1991). When the companion loses almost all of its envelope and mass transfer ceases, the endpoint of the evolution is a circular binary with a weak field MSP NS and an He or CO WD, the remaining core of the companion. A fast-growing population of eccentric $(e>0.5)$ PSR J1748-2021B. Binary MSPs have been recently revealed in globular clusters (Freire et al. 2007). Note that the formation of an LMXB invokes a binary evolution in which the progenitor system has to lose $90 \%$ of its initial mass and up to $99 \%$ of its initial orbital angular momentum (van den Heuvel 2009). 


\section{Table 1 List of some Massive CVs. ${ }^{*}$}

\begin{tabular}{lccc}
\hline \hline Name & $M_{\odot}$ & $P_{\text {orb }}$ & $M_{1} / M_{2}$ \\
\hline CAL 83 & 1.3 & 1.047 & - \\
CI Aql & 1.2 & 0.618 & 0.8 \\
BV Cen & 1.24 & 0.611 & 1.12 \\
RU Peg & 1.21 & 0.374 & 1.29 \\
MU Cen & 1.2 & 0.342 & 1.2 \\
BF Eri & 1.28 & 0.270 & 2.3 \\
VY Scl & 1.22 & 0.232 & 2.7 \\
BD Pav & 1.15 & 0.179 & 1.5 \\
U Gem & 1.2 & 0.176 & 2.6 \\
CU Vel & 1.23 & 0.078 & 5.0 \\
DP Leo & 1.2 & 0.062 & 5.5 \\
IP Peg & 1.16 & 0.16 & 2.08 \\
RX And & 1.14 & 0.209 & 2.4 \\
EY Cyg & 1.1 & 0.49 & 2.27 \\
WW Hor & 1.1 & 0.08 & 5.8 \\
SS Aur & 1.08 & 0.182 & 2.8 \\
\hline *The data are taken firn
\end{tabular}

${ }^{*}$ The data are taken from Ritter \& Kolb (2011).

Table 2 Descriptive statistical data for histograms of orbital period.

\begin{tabular}{lccccccc}
\hline \hline Name & Total number & Mean & Stand. Dev. & Sum & Mini & Median & Max. \\
\hline CVs & 178 & -0.916 & 0.255 & -163.11 & -1.267 & -0.894 & 0.30 \\
LMXBs & 36 & -0.835 & 0.655 & -30.054 & -1.555 & -0.80 & 1.073 \\
MSP (Galactic disk) & 91 & -0.356 & 0.242 & -214.39 & -2.807 & -2.405 & -1.713 \\
MSP (GC) & 64 & -0.053 & 0.822 & -3.45 & -1.179 & -0.159 & 2.282 \\
\hline
\end{tabular}

Table 3 Descriptive statistical data for histograms of spin period .

\begin{tabular}{lccccccc}
\hline \hline Name & Total number & Mean & Stand. Dev. & Sum & Mini & Median & Max. \\
\hline CVs & 120 & 3.53 & 0.527 & 621.133 & 1.755 & 3.718 & 4.458 \\
LMXBs & 37 & -1.978 & 1.215 & -73.201 & -2.79 & -2.497 & 2.057 \\
MSP (Galactic disk) & 91 & -2.356 & 0.242 & -214.39 & -2.807 & -2.405 & -1.713 \\
MSP (GC) & 120 & -2.37 & 0.21 & -283.39 & -2.86 & -2.38 & -1.76 \\
\hline
\end{tabular}

\subsection{Accretion Induced Collapse of white dwarfs}

Despite there being a clear-cut theoretical prediction of Accretion Induced Collapse (AIC) to form MSPs (Canal \& Schatzman 1967; Nomoto 1984, 1987), only recently have transient surveys had sufficient depth field of view to potentially detect and characterize these events through the optical and infrared light curves and spectra for the Nickel-rich outflows (Metzger et al. 2009ab \& and Darbha et al. 2010). However not all compositions can be involved in AIC. A key factor in the choice is the density of the chemical composition of the system (Isern \& Hernanz 1994).

In C-O WDs, the high temperature and great density can produced ignition. Consequently, the thermonuclear explosion causes the nuclear burning shell to be ejected leading to disruption of the WD and explosion as an SN Ia with a rate $10^{-4} \mathrm{yr}^{-1}$ (Popov \& Prokhorov 2007). This event can help us to possibly measure distances and the cosmological constant accurately (Bradely 2010). However, CVs may have implications for the still open question of which objects actually produce $\mathrm{SNe} \mathrm{Ia}$, owing to their long formation times and their relatively low accretion rates (Zortovic et al. 2010).

The scenario for an O-Ne-Mg WD is begin once the nuclear reaction starts at the center, the burning propagates throughout the entire star, then the ignition can happen in the interior. The burning which propagates outward makes central temperatures and pressures high enough to lead to a collapse of the WD, and induces the contraction of the star. This continues until it reaches the Chandrasekhar limit, and finally the star collapses homogeneously leading to the formation of an NS. Consequently, the AIC can be a significant contribution to the total number and evolution of LMXB (van den Heuvel 1997); notice that the minimum density for obtaining a collapse is $5.5 \times 10^{9} \mathrm{~g} / \mathrm{cm}^{3}$.

Depending on the strength of the WDs magnetic field, the matter flowing through the Lagrangian point can form 
either a full or partial accretion disk or else it will follow the magnetic field lines down to the surface at the magnetic poles (Warner 1995). It is therefore important to monitor long-period CV systems in order to gain insight into the dynamics and binary evolution in those systems most likely to undergo AIC.

At present, it is a well established fact that low mass WD stars should be formed during the evolution in close binary systems. Here the orbital evolution of these binaries, and the mass-transfer rate is driven by magnetic braking of the secondary for long-period systems, as well as by gravitational radiation for short-period systems (Beuermann \& Riensch 2002). This situation is in agreement with the possibility to apply the AIC route in binaries to produce binary MSPs. It occurs either because the WD began accreting as an $\mathrm{ONeMg}$ or because the accretion rate onto the WD does not allow them to remain a C-O WD. The AIC formation channel of MSP evolution is depicted in Fig. 1

\section{The Corbet diagram}

Various aspects of the statistical work performed by distributions for orbital-period and spin-period elucidate the origin and the mechanisms responsible for MSP formation. The Corbet diagram refers to the $P_{\text {spin }}-P_{\text {orb }}$ relation, proposed first by Corbet $(1984,1985)$, who noted there is a $P_{\text {spin }} \alpha P_{\text {orb }}^{2}$ correlation for Be systems, whereas $P_{\text {spin }} \alpha P_{\text {orb }}^{4 / 7}$ for systems with radially expanding winds was soon identified by van den Heuvel \& Rappaport (1987) (Norton et al. 2004; Yan et al. 2010). Fig. 2 shows the four types of binaries, which represent a panoramic perspective of binaries. The LMXBs (white circles) have short orbital and spin periods. It can be seen clearly that the CVs (black squares) are almost all spread at the top left corner, with orbital periods that typically range from approximately 0.06 to 2 days, and long spin periods (slow rotators). That means in the case of a massive $\mathrm{CV}$, if it accretes enough matter from the companion till it reaches the Chandrasekhar limit, it will collapse to become an NS during the AIC producing a short period binary MSP.

It should be noticed that at the moment no model is able to reproduce all of the observed binary properties; in particular, the predicted distributions of mass, orbital period and mass ratio tend not to match the observations very well (Reggiani \& Meyer 2011). If a model can be described by the correlation between these objects, many more CVs would be contributed as good progenitors of MSPs. This can be readily shown via a Venn diagram in Fig. 3, which shows how four sets can intersect. However observations of long orbital periods, more massive CVs and LMXBs in disparate environments can at least put constraints on theoretical binary formation models (Norton et al. 2004).

Our list of CVs is drawn from the new catalogue of Ritter \& Kolb (2011), as a starting point. The number of CVs shows that a few sources are massive $\sim M \geq 1.1 M_{\odot}$ (see Table 3). Fig. 4 shows the Gaussian distrbution of massive

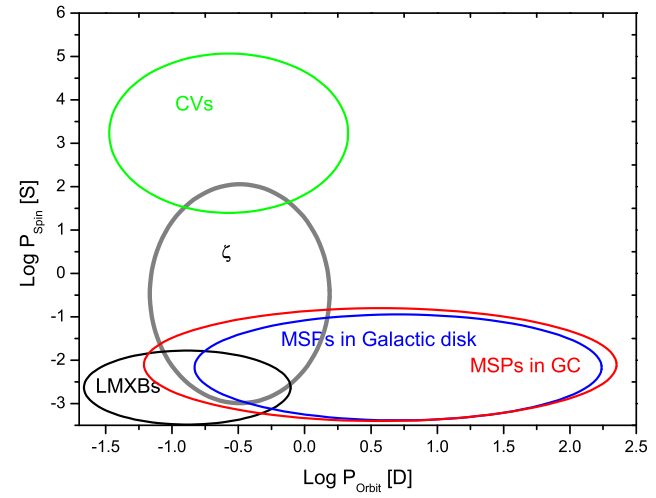

Fig. 3 A Venn diagram showing the relation among four different kinds of objects: MSPs in Galactic disk, MSPs in globular cluster, LMXBs and CVs. $\zeta$ represents the model.

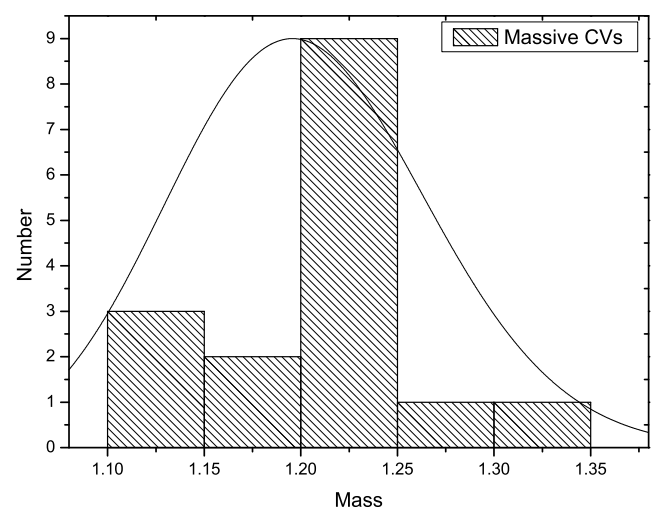

Fig. 4 Mass distribution of the observed sample of massive CVs. The solid line is the curve fitted using a Gaussian function. Data from Ritter \& Kolb (2011) were used to construct this distribution.

$\mathrm{CVs}$, with maximum at $M_{W D} \sim 1.3 M \odot$, where the mean mass of these sources is $M_{W D}=1.19 \pm 0.15 M \odot$. On the other hand, long orbital periods in the MSP distribution can be elucidated by the significant imparted kicks to the companion during the SN Ia. The kick speed, $V_{k i c k}$, is modelled by a Maxwellian distribution, as given by Hansen \& Phinney (1997) with a dispersion $V \sigma=190 \mathrm{Kms}^{-1}$

$$
P\left(V_{k i c k}\right)=\sqrt{\frac{2}{\pi}} \frac{V_{k i c k}^{2}}{V_{\sigma}^{3}} e^{-\frac{V_{k i c k}^{2}}{2 V_{\sigma}^{2}}}
$$

If the binary orbit is disrupted, this leaves a single MSP. This result agrees with the model by Podsiadlowski et al. (2002) and Taam (2004). The MSPs occupy a region in this diagram much larger than that of the LMXBs, and the process concurs with an evolutionary scenario of a binary MSP.

In order to investigate the distribution of two observed quantities for the four kinds of astronomical objects more clearly, we produced two histograms (see Figs. 3 \& 4) for the distribution of orbital and spin periods. Fig. 3 shows relatively Gaussian and regular distributions for most objects. A large fraction of these systems has an orbital period ranging between 40 minutes to 2 days, except for a few MSPs 
Table 4 Parameters of binary systems with compact companions.

\begin{tabular}{|c|c|c|c|c|c|c|c|}
\hline Group & Sub-group & Obser. evid. & $\begin{array}{l}P_{\text {orb }} \\
(\mathrm{D})\end{array}$ & $\begin{array}{l}P_{s} \\
(\mathrm{~S})\end{array}$ & $\begin{array}{l}M_{c} \\
M_{\odot} \\
\end{array}$ & $\begin{array}{c}B \\
(\mathrm{G}) \\
\end{array}$ & Ref. \\
\hline High-mass companion & $\begin{array}{c}\mathrm{NS}+\mathrm{NS} \\
\mathrm{NS}+(\mathrm{ONeMg}) \mathrm{WD} \\
\mathrm{NS}+(\mathrm{CO}) \mathrm{WD} \\
\end{array}$ & $\begin{array}{l}\text { PSR B1913+16 } \\
\text { PSR J1435-61 } \\
\text { PSR J1802-21 } \\
\end{array}$ & $\begin{array}{c}0.32 \\
1.35 \\
0.7 \\
\end{array}$ & $\begin{array}{l}0.059 \\
0.093 \\
0.012 \\
\end{array}$ & $\begin{array}{c}1.4398 \\
<1.75 \\
0.9\end{array}$ & $\begin{array}{c}10^{10 i} \\
5 \times 10^{8} \\
9.7 \times 10^{8} \\
\end{array}$ & $\begin{array}{l}1 \\
2 \\
3\end{array}$ \\
\hline Low-mass companion & $\mathrm{NS}+(\mathrm{He}) \mathrm{WD}$ & $\begin{array}{l}\text { PSR 0437-47 } \\
\text { PSR J1744-39 } \\
\end{array}$ & $\begin{array}{l}5.74 \\
0.19 \\
\end{array}$ & $\begin{array}{l}0.005 \\
0.172 \\
\end{array}$ & $\begin{array}{c}0.2 \\
0.08 \\
\end{array}$ & $\begin{array}{l}3 \times 10^{8} i i \\
1.7 \times 10^{10} \\
\end{array}$ & $\begin{array}{l}4 \\
5\end{array}$ \\
\hline $\begin{array}{l}\text { Non-recycled pulsar } \\
\text { Un-evolved Companion }\end{array}$ & $\begin{array}{l}\mathrm{NS}+(\mathrm{CO}) \mathrm{WD} \\
\text { Non-degenerate } \\
\end{array}$ & $\begin{array}{l}\text { PSR J0621+10 } \\
\text { PSR 1259-63 } \\
\end{array}$ & $\begin{array}{c}8.32 \\
0.047 \\
\end{array}$ & $\begin{array}{c}0.028 \\
1236.9 \\
\end{array}$ & $\begin{array}{c}0.75 \\
30 \\
\end{array}$ & $\begin{aligned} 1.2 & \times 10^{9} i i i \\
3 & \times 10^{11}\end{aligned}$ & $\begin{array}{l}6 \\
7\end{array}$ \\
\hline Millisecond Pulsars & $\begin{array}{c}\text { Millisecond companion } \\
\text { X-ray transients }\end{array}$ & $\begin{array}{r}\text { PSR J1903+032 } \\
\text { IGR J00291+59 }\end{array}$ & $\begin{array}{l}0.002 \\
0.102\end{array}$ & $\begin{array}{l}0.015 \\
0.001 \\
\end{array}$ & $\begin{array}{c}\sim 0.15-0.45 \\
\quad<1.6\end{array}$ & $\begin{array}{l}10^{8}-10^{9} \\
3 \times 10^{8} \text { iv } \\
\end{array}$ & $\begin{array}{l}8 \\
9\end{array}$ \\
\hline Cataclysmic Variables & $(\mathrm{CO}) \mathrm{WD}+(\mathrm{He}) \mathrm{WD}$ & AM Her. & 0.139 & 11960 & 0.76 & $10-8010^{8 v}$ & 10 \\
\hline
\end{tabular}

${ }^{1}$ Weisberg et al. 2010, ${ }^{2}$ Jacoby et al. 2006, ${ }^{3}$ Ferdman et al. 2010, ${ }^{4}$ Breton et al. 2007, ${ }^{5}$ van Kerkwijk et al 2005, ${ }^{6}$ Ray et al. $1992,{ }^{7}$ : Liu \& Li 2009, ${ }^{8}$ Marco et al. 1997, ${ }^{9}$ van den Heuvel 2009, ${ }^{10}$ Ritter \& Kolb 2011.

${ }^{i}$ Jasinta et al. 2005, ${ }^{i i}$ Zavlin et al. 2002, ${ }^{i i i}$ Eric et al. 2002, ${ }^{i v}$ Papitto et al. 2006, ${ }^{v}$ Sion et al. 2006

Abbreviations: $P_{s}$ : Spin period in seconds. $P_{o r b}$ : Orbital period in days. $M_{c}$ : Mass of companion in solar mass. Ref.: References for the listed parameters. $B$ : Magnetic field in Gauss. Obser. evid.: Observational evidence for each group.

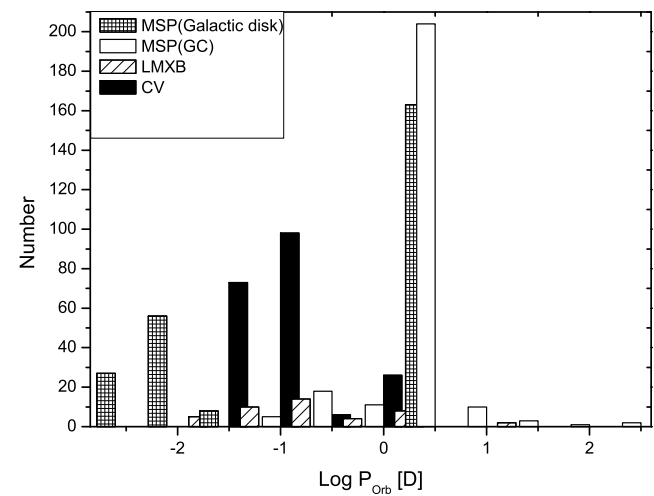

Fig.5 The distribution of orbital-periods for four different kinds of objects: MSPs in Galactic disk (dense histogram), MSPs in globular cluster (solid), LMXBs (sparse) and CVs (black).

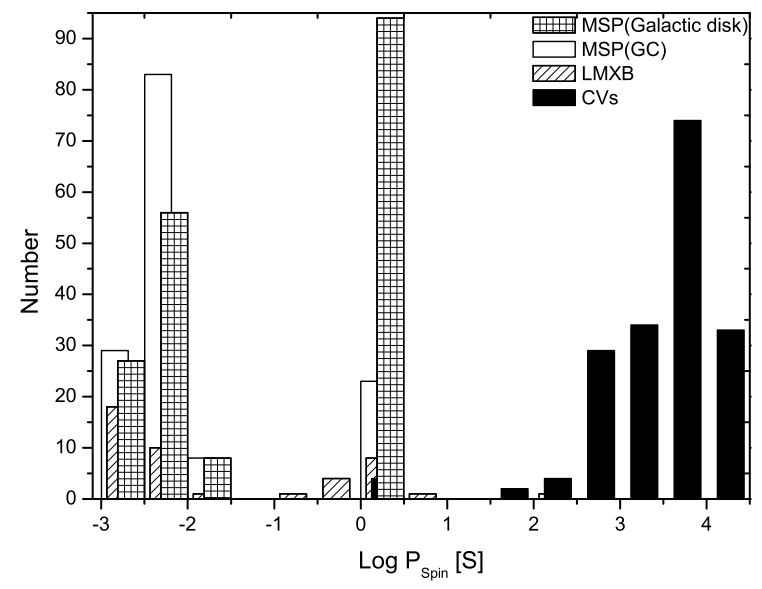

Fig. 6 The distribution of spin-periods for four different kinds of objects: MSPs in Galactic disk (dense histogram), MSPs in globular cluster (solid), LMXBs (sparse) and CVs ( black). sources in a globular cluster which have relatively longer orbital periods. It should be noticed that the data clearly suffer from selection effects, in particular the lack of many long orbital period systems such as CVs, that will ultimately determine the answer about the progenitors of MSPs.

Concerning the histogram for spin period (Fig. 4), we see that the distribution is almost bimodal with spin periods ranging from 30 seconds to 8 hours. The majority of spin periods for the MSPs population is in the sub-second region $\sim 20 \mathrm{~ms}$. It is interesting to note that few systems in globular clusters have spin periods shorter than those in the Galactic disk. This is may have due to a different formation mechanisms or shorter life-time to gravitational decay (Manchester 2006). There is a quite dramatic cut-off in the spin periods ranging from 7 to 40 seconds; which may be due to the sensitivity of the radio observation process and the selection effects. The statistical parameters for orbital and spin period histograms are listed in Table 2 and 3, respectively. It is noteworthy to mention here that if we apply the empirical formula of the Keplerian frequency, this leads to deriving the value of spin period for the MSP from WD as:

$$
\frac{P_{W D}}{P_{N S}} \sim\left(\frac{R_{W D}}{R_{N S}}\right)^{3 / 2} \sim 10^{4.5}
$$

where the minimum value of spin period for a WD is $P_{W D} \sim 30 s$. Therefore

$$
P_{N S} \sim 10^{-3} s
$$

can be considered as a secure range for the NS spin value. This clearly supports the view that the AIC effect can play an important role in deriving the spin period for an MSP. 


\section{Observational Data and Target Selection}

We gathered a relatively large number of observations for binary systems with compact companions from different catalogues. The sample contains a wide variety of sources, such as MSPs, LMXBs and CVs. It has two observed quantities: orbital period and spin period measurements.

The collected observations show that the number of MSPs listed in the ATNF (Australia Telescope National Facility) Pulsar Catalogue (Manchester et al. 2005) 1 is now close to 2000 pulsars; among them we have 211 MSPs, which is considered a relatively small number. 120 MSP sources are in globular clusters and 91 are in the galactic disk. Since MSPs in galactic disk and globular clusters may have different properties, we divided the sample into two groups. It should be noticed that the measurement for MSPs in the galactic disk is relatively accurate and reliable (Manchester 2006).

The data for CVs were distributed as follows:

- 100 objects from Lilia ferrario/ (private communications)

- 34 objects from the Koji Mukai IP page2,

- 52 objects were added from (Ritter \& Kolb 2011), Cataclysmic Binaries, Low-Mass X-Ray Binaries and Related Objects 3 .

- One IP source, V2069 = IGR J21237 (Martino et al. 2009)

The data of LMXBs were bifurcated into two main catalogues:

- 25 objects; Cataclysmic Binaries, Low-Mass X-Ray Binaries and Related Objects ${ }^{1}$, (Ritter \& Kolb 2011).

- 12 objects from the catalogue of low-mass X-ray binaries in the Galaxy, LMC and SMC (Fourth edition) (Liu et al. 2007).

Table 4 summarizes the parameters of some compact companion binary systems with a number of different physical characteristics.

\section{Summary and Conclusions}

The primary goal of this work is to gather in a single place an updated list of different compact objects, to be used for tests against various theoretical predictions of characteristics of MSPs progenitors. Several conclusions can potentially be derived from this work

1. Based upon updated data and a large number of objects, the analyzed distribution of the observed sample indicates revisions and refinement for the evolution of binary MSPs and their progenitors. This highlights the contribution value of studying the Corbet diagram (similar to the H-R or B-P diagrams) to understand evolution and formation mechanism events for different binaries.

\footnotetext{
${ }^{1} \mathrm{http} / / /$ www.atnf.csiro.au/research/pulsar/psrcat/.html

2 http://asd.gsfc.nasa.gov/Koji.Mukai/iphome/systems/.html

3 http://www.mpa-garching.mpg.de/RKcat/
}

2. The recycling scenario proved our extensive sample for the short orbital period of such binaries and very small companion masses.

3. The AIC scenario is potentially significant, and it remains to be determined whether alternative evolutionary scenarios need to be invoked. Further work will certainly clarify this issue as known sources are better characterized.

4. Our results show that massive CVs increase the proportion of progenitors for binary MSPs with short orbital periods ( $<2$ hours). This result agrees with some theoretical predictions such as those by Podsiadlowski et al. (2002) and Taam (2004).

5. Massive CVs predict a significant fraction of progenitors of present day binary MSP (i.e. CAL 83 and CAL 87). If matter is accreted at a rate of $M \sim 10^{16} \mathrm{gs}^{-1}$, and the total mass accreted exceeds a critical value $\Delta M_{\text {crit }} \sim$ $0.1-0.2 M_{\odot}$, then it will be recycled to become an MSP after the mass reaches the Chandrasekhar limit during the AIC process.

6. An alternative scenario that depends on a definite chemical composition, is a result of an accreting C-O WD; at such low mass transfer rates, the nuclear burning of the hydrogen accreted on the surface of the WD takes place in the form of an SN Ia, in which most or all of the accreted matter is ejected, consequently imparting significant kicks to the companion creating long orbital periods. In some cases this process disrupts the system and creates a single MSP.

7. The long orbital periods occur for half of the MSP population, depending on their mass ratio (q) according to

$$
q \begin{cases}M_{c}>M_{M S P}, & \text { Short orbit } \\ M_{c}<M_{M S P}, & \text { Long orbit }\end{cases}
$$

Once mass transfer starts, the loss of angular momentum causes the orbit to shrink, and thus the orbital period becomes shorter. In the case of CVs, the mass of the companion is less than the mass of $\mathrm{CVs}$, therefore, all these systems appear in a close orbit scale.

8. The LMXBs appear to have orbital periods shorter than entire MSP population. This clearly supports the view that LMXB progenitors must satisfy a large number of evolutionary and structural constraints of MSPs. On the other hand, the LMXBs population has instigated active discussion about the possibility that at least some of them originate from AIC. Probably, the process will make sense in the evolutionary scenario of binary MSPs or other mechanisms we have not encountered yet. There maybe an otherwise undetectable fraction of X-ray MSPs since all pulsar surveys have some limiting flux density. Future work will go steps further, using the more extensive data set now available from Ritter \& Holb (2011) and other sources.

9. Further binary studies and massive CVs and long orbital periods are needed to study the dependence of the $P_{\text {Spin }}-P_{\text {orb }}$ relation on dynamical processes and to test possible variations in the orbital periods, spin peri- 
ods and the mass ratio distribution of different formation mechanisms. Therefore, there is still a debate about how to construct a unified model to explain the evolution of LMXB, CVs and MSPs. How does the process of dynamical AIC collapse usually gets started. And there are related debates over what processes are principally responsible for isolated MSPs.

Finally, however the current available data for CVs are not sufficiently accurate or numerous to allow precise analysis. But we hope that the results of this work will constitute a base for further studies on the observational properties of massive CVs and long orbital periods.

\section{Acknowledgements}

We are grateful for the discussions with Edward van den Heuvel, and Andrew Norton. The authors would like to thank Lilia Ferrario for AM Hers data. The research presented here made an extensive use of the 2011 version of the ATNF Pulsar Catalogue (Manchester et al. 2005). We also thank the anonymous referee for a careful reading of our manuscript and for numerous useful comments. This research has been supported by NSFC (No.10773017) and National Basic Research Program of China (2009CB824800).

\section{References}

Alpar, M. A., Cheng, A. F. \& Ruderman, M. A. et al.: 1982, Nature 300,728

Beuermann, K. \& Riensch, K.: 2002, A\&A 381, 487

Bhattacharya, D. \& van den Heuvel, E. P. J.: 1991, Phys. Rep. 203

Bhattacharya, D.: 1996, ASPC 105, 547

Bradely, E.: 2010, ApJ Supp. 187, 275

Breton R. P., Roberts M. S. E. \& Ransom, S. M. et al. 2007, ApJ, 661,1073

Burderi, L., di Salvo, T. \& Lavagetto, G. et al.: 2007, ApJ 657, 961

Camilo, F., Lyne, A. G. \& Manchester, R. N. et al.: 2001, ApJ 548, 187

Canal, R. \& Schatzman, E.: 1967, Ast. Ap. 46, 229

Champion, D. J., et al.: 2008, Sci, 320, 1309

Corbet R H D. 1984, Astron Astrophys, 141, 91

Corbet R H D. 1985, Space Sci Res, 40, 409

Darbha, S., Metzger, B. D. \& Quataert, E. et al.: 2010 MNRAS, 409,846

Edelstein, J., Foster, R. S. \& Bowyer ,S.: 1995, ApJ 454, 442

Ferdman, R. D., Stairs, I. H. \& Kramer, M. et al.: 2010, ApJ711, 764

Ferrario, L. \& Wickramasinghe, D. T.: 2007a, AIP Con. 968, 188

Ferrario, L. \& Wickramasinghe, D. T.: 2007b, AIP Conf. 968, 194

Hansen, B. M. S., \& Phinney, E. S.: 1997, MNRAS 291, 569

Hurley, J. R., Tout, C. A. \& Wickramasinghe, D. T. et al.: 2010, MNRAS 402, 1437

Isern, J. \& Hernanz, M.: 1994, MmSAI 65, 339

Ivanova, N., Heinke, C.O. \& Rasio, F.A. al.: 2008, MNRAS 386, 553

Jasinta, D., Dewi, M. \& van den Heuvel, E. P. J.: ASP Conf. Ser. 328, 2005

Jacoby, B. A., Chakrabarty, D. \& van Kerkwijk, M. H. et al.: 2006, ApJ 640, 183
Li, X.D.: 2010, Sience in China 53, 9

Liu, X-W. \& Li, X-D.: 2009, ApJ 692, 723

Liu, Q. Z., van Paradijs, J. \& van den Heuvel, E P J.: 2007, A\&A 469, 807

Liu, Q. Z et al. 2011, MNRAS, 415, 3349

Lorimer, D. R. 2009, Neutron Stars and Pulsars, ed. W. Becker (Astrophysics and Space Science Library, Vol. 357; Berlin: Springer)

Lyne, A. G., Burgay, M. \& Kramer, M. et al.:2004, Science 303, 1153

Manchester, R. N., Hobbs, G. B. \& Teoh, A. et al.: 2005, AJ 129, 1993

Manchester, R. N. 2006, Advances in Space Research, 38, 2709

Martino, D., Bonnet-Bidaud, J. M. \& Falanga, M. et al.: 2009, ATel 2089.1D

Metzger, B. D., Piro, A. \& Quataert, E.: 2009a. MNRAS 396, 304

Metzger, B. D., Piro, A., Quataert, E. \& Thompson, T.: 2009b. MNRAS 396, 1659

Nomoto, K.: 1984, ApJ 277, 791

Nomoto, K.: 1987, ApJ 322, 206

Norton A. J., Wynn G. A. \& Somerscales R. V.:2004. ApJ, 614, 349

Papitto, A. Riggio, L. Burderi, et al.: 2011, A\&A 528, A55

Podsiadlowski, Ph., Rappaport, S. \& Pfahl, E.: 2002. ApJ 565, 1107

Popov, S .B \& Prokhorov, M. E.: 2007, Phys.-Usp. 501123

Ray, P. S., Grove, J. E. \& Kurfess, J. D. et al.: 1992, AIP Conf. Proc. 280, 249

Reggian M. \& Meyer M.: 2011, ApJ, 738, 60

Ritter, H. \& Kolb, U.: 2011, Catalogue of Cataclysmic Binaries, Low-Mass X-Ray Binaries and Related Objects, http://www.mpa-garching.mpg.de/RKcat/cbcat

Romani, G. M.: 1990, Nature 347,741

Sutantyo, W. \& Li, X.D.: 2000, A\&A 360, 633

Sion, E. M., Solheim, J.E. \& Szkody, P. et al.: 2006, astro-ph/0602119 v1

Srinivasan, G., van den Heuvel, E.P. J.: 1982, Astro. Astrophys. 108, 143

Taam, R. E.: 2004, RevMexAA 20, 81 Cambridge: Cambridge University Press, 1987. 291

van den Heuvel E. P. J. 2004, Science 303, 1143

van den Heuvel E. P. J \& Rappaport S. 1987, Cambridge: Cambridge University Press, 291

van den Heuvel E. P. J., 2009, Physics of Relativistic Objects in Compact Binaries: From Birth to Coalescence, Astrophysics and Space Science Library, 359, p. 125

Warner, B.: 1995, ASP Conf. Ser. 85, 343

Weisberg ,J. M., Nice, D. J., \& Taylor, J. H.: 2010, ApJ 722, 1030

Wickramasinghe D. T., Hurley J. R., Ferrario L., Tout C. A. \& Kiel P. D. 2009, JPhCS., 172, 2037

Yan, Y., Esamdin, A. \& Yin, H. X.: 2010, Sience in China 53, 9

Zavlin, G., Pavlov, D., \& Sanwal A. et al.: 2002, ApJ 569, 894902,

Zortovic M., Schreiber M. R., Gänsicke B. T. \& Nebot GómezMorán A. 2010, A\&A, 520, A86 